\title{
SPL Flavor Terminology
}

National Cancer Institute

\section{Source}

National Cancer Institute. SPL Flavor Terminology. NCI Thesaurus. Code C73339.

Terminology used for the representation of the information on a pharmaceutical product the most predominant flavor in the framework of the Structured Product Labeling documents. 